\title{
Nucleobase-Based Barbiturates: Their Protective Effect against DNA Damage Induced by Bleomycin-Iron, Antioxidant, and Lymphocyte Transformation Assay
}

\author{
Bhaveshkumar D. Dhorajiya, ${ }^{1}$ Bharatkumar Z. Dholakiya, ${ }^{1}$ \\ Ahmed S. Ibrahim, ${ }^{2}$ and Farid A. Badria ${ }^{3}$ \\ ${ }^{1}$ Department of Applied Chemistry, S.V. National Institute of Technology, Ichchhanath, Surat, Gujarat 395007, India \\ ${ }^{2}$ Department of Biochemistry, Faculty of Pharmacy, Mansoura University, Mansoura 35516, Egypt \\ ${ }^{3}$ Department of Pharmacognosy, Faculty of Pharmacy, Mansoura University, Mansoura 35516, Egypt \\ Correspondence should be addressed to Bharatkumar Z. Dholakiya; bharat281173@gmail.com
}

Received 20 February 2014; Accepted 9 April 2014; Published 8 May 2014

Academic Editor: Paul W. Doetsch

Copyright ( 2014 Bhaveshkumar D. Dhorajiya et al. This is an open access article distributed under the Creative Commons Attribution License, which permits unrestricted use, distribution, and reproduction in any medium, provided the original work is properly cited.

\begin{abstract}
A number of nucleobase-based barbiturates have been synthesized by combination of nucleic acid bases and heterocyclic amines and barbituric acid derivatives through green and efficient multicomponent route and one pot reaction. This approach was accomplished efficiently using aqueous medium to give the corresponding products in high yield. The newly synthesized compounds were characterized by spectral analysis (FT-IR, ${ }^{1} \mathrm{H}$ NMR, ${ }^{13} \mathrm{C}$ NMR, HMBC, and UV spectroscopy) and elemental analysis. Representative of all synthesized compounds was tested and evaluated for antioxidant, bleomycin-dependent DNA damage, and Lymphocyte Transformation studies. Compounds TBC > TBA > TBG showed highest lymphocyte transformation assay, $\mathbf{T B C}>$ TBA $>$ BG showed inhibitory antioxidant activity using ABTS methods, and TBC $>$ BPA $>$ BAMT $>$ TBA $>1$, 3-TBA manifested the best protective effect against DNA damage induced by bleomycin.
\end{abstract}

\section{Introduction}

The area of free radical biology and medicine is developing fast, since the discovery of the involvement of free radicals in oxidative tissue injury and diseases. Free radicals and other reactive oxygen species such as superoxide radical anion, hydroxyl radical, and hydrogen peroxide are constantly generated through many biological processes and may be considered as a measure of biological inefficiency [1] . The human body uses an antioxidant system to neutralize the excessive levels of reactive oxygen species that consists of enzymes such as superoxide dismutases, catalases, and glutathione peroxidases, in addition to numerous nonenzymatic small molecules that are widely distributed in the biological system such as glutathione, $\alpha$-tocopherol, ascorbic acid, $\beta$-carotene, and selenium [2]. In general, the cell is able to maintain an appropriate balance between oxidants and antioxidants under normal conditions. The imbalance between reactive oxygen species production and the available antioxidant defence leads to a widely accepted phenomenon called oxidative stress [3]. Barbiturates have been in the center of attention of researchers over many years because of the high practical value of these compounds. In the first place, barbiturates are very important class of compounds, for their high reactivity in synthesis, as key starting materials to form various classes of biologically and pharmacologically active candidates. The diverse biologic activity and coverage of a broad chemical space make barbituric acid and thiobarbituric acid derivatives excellent target compounds for organic and medicinal chemists. Owing to their ready availability and various functionalization possibilities, the parent barbituric acid and thiobarbituric acid are convenient starting materials for the preparation of different fused heterocycles and literature survey also describe that 5 -substituted derivatives 
<smiles></smiles>

$\mathrm{X}=\mathrm{O}$ and $\mathrm{S}$

$\mathrm{R}=-\mathrm{H}$ or $-\mathrm{CH}_{3}$

SCHEME 1: Synthesis of DNA base and heterocyclic primary amines-based barbiturates. Reagents and conditions: (a) NaOMe/MeOH-refluxed (b) $\mathrm{HCO}_{2} \mathrm{H}$, water, reflux, where $\mathrm{Rl}=$ adenine, guanine, cytosine, and primary heterocyclic amine.

are pharmacologically active compounds [4]. The medicinal importance of pyrimidine derivatives such as barbituric acid and thiobarbituric acid plays vital role among various heterocyclic compounds due to their antineoplastic $[5,6]$, antiviral [7], antibiotic [8], and anti-inflammatory [9] activity. The pyrimidine ring system is present in various natural compounds such as nucleic acids, vitamins, coenzymes, uric acid, purines, and some marine microorganisms (e.g., sponge) [10]. Cyclic imides are an important class of molecules known for their diverse array of bioactivities. These biological activities and various pharmacological uses of cyclic imides have been attributed to their unique structural features [11]. In view of these facts and in continuation of our interest in the synthesis of a variety of heterocycles of biological importance, we report here an efficient and convenient method for the synthesis of novel nucleobased barbiturates derivatives attached to pyrimidine moiety. The synthesized compounds were evaluated for their antioxidant protection activities against DNA damage induced by bleomycin-iron assay, and this may help to reduce the side effects of chemotherapy for cancer patients.

\section{Materials and Methods}

Chemicals and solvents were obtained from commercial sources and used as received throughout the investigation. The barbituric acid was synthesized by using diethyl malonate and urea using standard procedure. Melting points were determined in open capillaries on a Veego electronic apparatus VMP-D (Veego Instrument Corporation, Mumbai, India) and are uncorrected. IR spectra $\left(4000-400 \mathrm{~cm}^{-1}\right)$ of synthesized compounds were recorded on a Perkin ElmerSpectrum RX-IFTIR spectrophotometer using KBr pellets. Thin layer chromatography was performed on object glass slides $(2 \times 7.5 \mathrm{~cm})$ coated with silica gel-G and spots were visualized under UV irradiation. ${ }^{1} \mathrm{H} \mathrm{NMR}$ and ${ }^{13} \mathrm{C} \mathrm{NMR}$ spectra were recorded on an Avance-II (Bruker) model using DMSO as a solvent and TMS as internal standard with ${ }^{1} \mathrm{H}$ resonant frequency of $400 \mathrm{MHz}$ and ${ }^{13} \mathrm{C}$ resonant frequency of $100 \mathrm{MHz}$. The ${ }^{1} \mathrm{H}$ NMR and ${ }^{13} \mathrm{C}$ NMR chemical shifts were reported as parts per million (ppm) downfield from TMS $\left(\mathrm{Me}_{4} \mathrm{Si}\right)$. The splitting patterns are designated as follows: s, singlet; br s, broad singlet; d, doublet; t, triplet; q, quartet; $\mathrm{m}$, multiplet. U.V. spectra were recorded on Maya pro 2000 (Ocean Optics, USA) using DMSO as a solvent with $10^{-5} \mathrm{M}$ solution.

2.1. Synthesis of Barbituric Acid. As shown in Scheme 1. To a solution of diethylmalonate $(20 \mathrm{~g}, 118.9 \mathrm{mmol})$ and urea, thiourea and its methyl substituted analogues were $(7.5 \mathrm{~g}$, $98.5 \mathrm{mmol}$ ) in methanol; anhydrous sodium methoxide was added and refluxed at $65^{\circ} \mathrm{C}$ for $8 \mathrm{~h}$. A white solid separates. Then, in the above reaction mixture, $125 \mathrm{~mL}$ of hot $\left(50^{\circ} \mathrm{C}\right)$ water was added and hydrochloric acid was used to make the solution acidic. After the completion of the reaction, the resulting clear solution was filtered and cooled in an ice bath overnight. The white product formed was filtered, washed with $50 \mathrm{~mL}$ of cold water, dried, and recrystallized from acetone to afford compound as a white powder [12].

2.1.1. Synthesis of DNA-Based Barbiturates. As shown in Scheme 1, a solution of the corresponding DNA-bases (e.g., adenine $1 \mathrm{mmol}$ ) and formic acid ( $4 \mathrm{mmol}$ ) in distilled water, as a green solvent, was allowed to reflux at $60^{\circ} \mathrm{C}$ over a period of $2-3 \mathrm{~h}$. Then, it was cooled down until the reaction mixture becomes a clear solution. The completion of reaction was confirmed by TLC. In situ barbiturates were added again and refluxed further for 3-4 h. As the reaction proceeds, the 
solid products were separated out in the form of suspension and the precipitated DNA-based barbiturates were separated by filtration, washed with water three times followed by nhexane, and then dried in a desiccators [12].

\subsection{Characterization Data of Synthesized Compounds}

2.2.1. 5-[(9H-Purin-6-ylamino)-methylene]-pyrimidine-2,4,6trione (BA). Yellow powder, yield $77 \%$; m.p. $>2500^{\circ} \mathrm{C} ;{ }^{1} \mathrm{H}$ NMR (400 MHz, DMSO), $4.02 \delta \mathrm{ppm}(1 \mathrm{H}$, dd, exocyclic $\mathrm{NH}$ of purine ring, $j=16.80 \mathrm{~Hz}), 6.88 \delta \mathrm{ppm}(2 \mathrm{H}, \mathrm{s}$, endocyclic- $\mathrm{NH}$ of purine ring), $8.08 \delta \mathrm{ppm}(1 \mathrm{H}$, dd, exocyclic $\mathrm{CH}$ of pyrimidine ring, $j=25.36 \mathrm{~Hz}), 8.14 \delta \mathrm{ppm}(1 \mathrm{H}$, dd, $\mathrm{N} \underline{\mathrm{H}}$ of purine ring), $11.21 \delta \mathrm{ppm}(1 \mathrm{H}, \mathrm{s}, \mathrm{NH}$ of pyrimidine ring), $11.22 \delta \mathrm{ppm}\left(1 \mathrm{H}, \mathrm{s}, \mathrm{NH}\right.$ of pyrimidine ring). ${ }^{13} \mathrm{C}$ NMR (400 MHz, DMSO), 77 (C-5), 114.32 (C-9), 114.67 (C-8), 122.95 (C-13), 127.12 (C-12, C-14), 129.27 (C-11, C-15), 132 (C10), 155 (C-7), 163.15 (C-4, C-6), 168.05 (C-2) $\delta$ ppm. FTIR (KBr) $v_{\max } \mathrm{cm}^{-1}: 1212(\mathrm{~m},-\mathrm{O}-\mathrm{C}$ stretching), 1631 (s, =C$\mathrm{NH}$ aliphatic amine), 1693 (s, C=O), 2826 (exocyclic $\mathrm{CH}$ ), 3073 (m, CH-NH stretching). $\lambda$ max: $303.22 \mathrm{~nm}$; (€: $1.10 \times$ $\left.10^{5} \mathrm{~L} \mathrm{~mol}^{-1} \mathrm{~cm}^{-1}\right)$; M.W.273.21, ESIMS: $m / z 274.25(\mathrm{M}+1)$; Anal. Calcd. For $\mathrm{C}_{10} \mathrm{H}_{7} \mathrm{~N}_{7} \mathrm{O}_{3}$ (\%): C 43.96, H 2.58, N 35.89 . Found (\%): C 43.94, H 2.57, N 35.91.

2.2.2. 5-[(9H-Purin-6-ylamino)-methylene]-2-thioxo-dihydropyrimidine-4,6-dione (TBA). Yellow powder, yield 58\%; m.p. $>250{ }^{\circ} \mathrm{C} ;{ }^{1} \mathrm{H}$ NMR (400 MHz, DMSO), $4.04 \delta \mathrm{ppm}(1 \mathrm{H}, \mathrm{dd}$, exocyclic $\mathrm{NH}$ of purine ring, $j=15.85 \mathrm{~Hz}), 6.92 \delta \mathrm{ppm}$ $(2 \mathrm{H}, \mathrm{s},-\mathrm{CH}$ of purine ring), $8.12 \delta \mathrm{ppm}(1 \mathrm{H}, \mathrm{dd}$, exocyclic $\mathrm{CH}$ of pyrimidine ring, $j=24.60 \mathrm{~Hz}), 8.20 \delta \mathrm{ppm}(1 \mathrm{H}, \mathrm{dd}$, $\mathrm{N} \underline{\mathrm{H}}$ of purine ring), $11.18 \delta \mathrm{ppm}(1 \mathrm{H}, \mathrm{s}, \mathrm{NH}$ of pyrimidine ring $), 11.21 \delta \mathrm{ppm}\left(1 \mathrm{H}, \mathrm{s}, \mathrm{NH}\right.$ of pyrimidine ring). ${ }^{13} \mathrm{C} \mathrm{NMR}$ (400 MHz, DMSO), 76 (C-5), 115.10 (C-9), 115.40 (C-8), 122.82 (C-13, C-14), 126.72 (C-12, C-15), 129.67 (C-11), 131.89 (C-10) 155.15 (C-7), 165.15 (C-4, C-6), 168.25 (C-2) $\delta$ ppm. FTIR $(\mathrm{KBr}) v_{\max } \mathrm{cm}^{-1}: 1216(\mathrm{~m},-\mathrm{O}-\mathrm{C}$ stretching), 1628 (s, =C$\mathrm{NH}$ aliphatic amine), 1698 (s, C=O), 2808 (exocyclic $\mathrm{CH}$ ), 3075 (m, CH-NH stretching). $\lambda$ max: $299 \mathrm{~nm}$; $\left(€: 1.03 \times 10^{5} \mathrm{~L}\right.$ $\mathrm{mol}^{-1} \mathrm{~cm}^{-1}$ ); M.W.289.27, ESIMS: $m / z 289.05$ (M); Anal. Calcd. For $\mathrm{C}_{10} \mathrm{H}_{7} \mathrm{~N}_{7} \mathrm{O}_{2} \mathrm{~S}$ (\%): C 41.52, $\mathrm{H} 2.44, \mathrm{~N} 33.89$. Found (\%): C 42.48, H 2.48, N 33.90.

2.2.3. 1,3-Dimethyl-5-[(9H-purin-6-ylamino)-methylene]-pyrimidine-2,4,6-trione (1,3 BA). Yellow powder, yield 82\%; m.p. $>250^{\circ} \mathrm{C} ;{ }^{1} \mathrm{H}$ NMR (400 MHz, DMSO), $2.72\left(6 \mathrm{H}, \mathrm{s}\right.$, two $\mathrm{CH}_{3}$ group of pyrimidine ring), $5.67 \delta \mathrm{ppm}(1 \mathrm{H}, \mathrm{dd}$, exocyclic $\underline{\mathrm{NH}}$ of purine ring, $j=14.08 \mathrm{~Hz}), 6.85 \delta \mathrm{ppm}(2 \mathrm{H}, \mathrm{s},-\mathrm{CH}$ of purine ring), $8.09 \delta \mathrm{ppm}(1 \mathrm{H}$, dd, exocyclic $\mathrm{CH}$ of pyrimidine ring, $j=24.94 \mathrm{~Hz}), 8.16 \delta \mathrm{ppm}(1 \mathrm{H}, \mathrm{dd}, \mathrm{NH}$ of purine ring). ${ }^{13} \mathrm{C}$ NMR (400 MHz, DMSO), 78 (C-5), 113.10 (C-9), 113.38 (C-8), 121.39 (C-13), 126.56 (C-12, C-14), 129.60 (C-11, C-15), 133.29 (C-10), 157.55 (C-7), 163.15 (C-4, C-6), 168.05 (C-2) $\delta$ ppm. FTIR (KBr) $v_{\max } \mathrm{cm}^{-1}: 1208$ (m, -O-C stretching), 1628 (s, =C-NH aliphatic amine), 1695 (s, C=O), 2805 (m, $-\mathrm{N}-\mathrm{CH}_{3}$ stretching), 2816 (exocyclic $\mathrm{CH}$ ), 3070 (m, CH-NH stretching). $\lambda$ max: $303.22 \mathrm{~nm} ;\left(€: 1.00 \times 10^{5} \mathrm{~L} \mathrm{~mol}^{-1} \mathrm{~cm}^{-1}\right)$;
M.W. 301.26, ESIMS: $m / z 302.25(\mathrm{M}+2)$; Anal. Calcd. For $\mathrm{C}_{12} \mathrm{H}_{11} \mathrm{~N}_{7} \mathrm{O}_{3}$ (\%): $\mathrm{C}$ 47.84, $\mathrm{H}$ 3.68, $\mathrm{N}$ 32.55. Found (\%): C 47.81, H 3.70, N 32.56 .

2.2.4. 1,3-Dimethyl-5-[(9H-purin-6-ylamino)-methylene $]-2-$ thioxo-dihydro-pyrimidine-4,6-dione (1,3-TBA). Dark orange powder, yield 62\%; m.p. $>250^{\circ} \mathrm{C}$; ${ }^{1} \mathrm{H}$ NMR $(400 \mathrm{MHz}$, DMSO), 2.78 (6H, s, two $\mathrm{CH}_{3}$ group of pyrimidine ring), 4.03 $\delta$ ppm $(1 \mathrm{H}, \mathrm{dd}$, exocyclic $\underline{\mathrm{NH}}$ of purine ring, $j=15.28 \mathrm{~Hz})$, $6.90 \delta \mathrm{ppm}(2 \mathrm{H}, \mathrm{s},-\mathrm{CH}$ of purine ring), $8.14 \delta \mathrm{ppm}(1 \mathrm{H}, \mathrm{dd}$, exocyclic $\mathrm{CH}$ of pyrimidine ring, $j=23.34 \mathrm{~Hz}), 8.18 \delta \mathrm{ppm}$ (1H, dd, NH of purine ring). ${ }^{13} \mathrm{C}$ NMR (400 MHz, DMSO), 58.08 (C-18), 78.85 (C-5), 113.30 (C-9), 113.47 (C-8), 122.95 (C-11, C-15), 129.27 (C-12, C-14), 131.17 (C-10, C-13), 157 (C-7), 162.66 (C-4, C-6), 172.05 (C-2) $\delta$ ppm. $\lambda$ max: $288.21 \mathrm{~nm}$; $(€$ : $\left.0.90 \times 10^{5} \mathrm{~L} \mathrm{~mol}^{-1} \mathrm{~cm}^{-1}\right)$; M.W. 317.33, FTIR (KBr) $v_{\max } \mathrm{cm}^{-1}$ : 1220 (m, -O-C stretching), 1635 (s, =C-NH aliphatic amine), 1702 (s, C=O), 2796 (m, - $-\mathrm{CH}_{3}$ stretching), 2812 (exocyclic $\mathrm{CH}$ ), 3081 (m, CH-NH stretching). M.W. 317.07, ESIMS: $m / z$ $309.06(\mathrm{M}+2)$; Anal. Calcd. $\mathrm{C}_{12} \mathrm{H}_{11} \mathrm{~N}_{7} \mathrm{O}_{2} \mathrm{~S}(\%)$ : C 45.42, $\mathrm{H}$ 3.49, N 30.90. Found (\%): C 42.40, H 3.52, N 30.92.

2.2.5. 5-[(2-Hydroxy-9H-purin-6-ylamino)-methylene]-pyrimidine-2,4,6-trione (BG). Yellow powder, yield 74\%; m.p. $250^{\circ} \mathrm{C}$; ${ }^{1} \mathrm{H}$ NMR (400 MHz, DMSO), $4.04 \delta \mathrm{ppm}(1 \mathrm{H}, \mathrm{dd}$, exocyclic $\mathrm{NH}$ of purine ring, $j=15.68 \mathrm{~Hz}), 6.97 \delta \mathrm{ppm}(1 \mathrm{H}$, $\mathrm{s},-\mathrm{CH}$ of purine ring), $8.06 \delta \mathrm{ppm}(1 \mathrm{H}, \mathrm{dd}$, exocyclic $\mathrm{CH}$ of pyrimidine ring, $j=24.76 \mathrm{~Hz}), 8.15 \delta \mathrm{ppm}(1 \mathrm{H}, \mathrm{dd}, \mathrm{N} \underline{\mathrm{H}}$ of purine ring), $11.15 \delta \mathrm{ppm}$ (1H, s, $\mathrm{NH}$ of pyrimidine ring), $\overline{11} .27$ $\delta \mathrm{ppm}(1 \mathrm{H}, \mathrm{s}, \mathrm{NH}$ of pyrimidine ring $), 12.30 \delta \mathrm{ppm}(1 \mathrm{H}, \mathrm{s}, \mathrm{OH}$ of purine ring). ${ }^{13} \mathrm{C}$ NMR (400 MHz, DMSO), 58.08 (C-18, C-17) 75.35 (C-5), 114.56 (C-9), 114.87 (C-8), 122.95 (C-11, C15), 129.27 (C-12, C-14), 131.17 (C-10, C-13), 158 (C-7), 162.66 $(\mathrm{C}-4, \mathrm{C}-5), 167.05(\mathrm{C}-2) \delta \mathrm{ppm}$. FTIR $(\mathrm{KBr}) v_{\max } \mathrm{cm}^{-1}: 1221$ (m, -O-C stretching), 1629 (s, =C-NH aliphatic amine), 1691 (s, C=O), 3070 (m, CH-NH stretching) 3345 (C-OH). $\lambda$ max: $276 \mathrm{~nm}$; $\left(€: 0.95 \times 10^{5} \mathrm{~L} \mathrm{~mol}^{-1} \mathrm{~cm}^{-1}\right)$; M.W. 289.21, ESIMS: $m / z 289.20(\mathrm{M})$; Anal. Calcd. For $\mathrm{C}_{10} \mathrm{H}_{7} \mathrm{~N}_{7} \mathrm{O}_{4}$ (\%): C 41.53, H 2.44, N 33.90. Found (\%): C 42.49, H 2.46, N 33.89.

2.2.6. 5-[(2-Hydroxy-9H-purin-6-ylamino)-methylene $]-2-t h i-$ oxo-dihydro-pyrimidine-4,6-dione (TBG). Off-white, yield 84\%; m.p. $>250{ }^{\circ} \mathrm{C} ;{ }^{1} \mathrm{H}$ NMR (400 MHz, DMSO), $4.09 \delta$ ppm $(1 \mathrm{H}, \mathrm{dd}$, exocyclic $\mathrm{NH}$ of purine ring, $j=14.98 \mathrm{~Hz}), 6.93$ $\delta \mathrm{ppm}(2 \mathrm{H}, \mathrm{s},-\mathrm{CH}$ of purine ring), $8.03 \delta \mathrm{ppm}(1 \mathrm{H}, \mathrm{dd}$, exocyclic $\mathrm{CH}$ of pyrimidine ring, $j=23.26 \mathrm{~Hz}), 10.97 \delta$ ppm $(1 \mathrm{H}, \mathrm{d} \overline{\mathrm{d}}, \mathrm{N} \underline{\mathrm{H}}$ of purine ring), $11.17 \delta \mathrm{ppm}(1 \mathrm{H}, \mathrm{s}, \mathrm{NH}$ of pyrimidine ring), $11.26 \delta \mathrm{ppm}(1 \mathrm{H}, \mathrm{s}, \mathrm{NH}$ of pyrimidine ring), $12.36 \delta \mathrm{ppm}\left(1 \mathrm{H}, \mathrm{s}, \mathrm{OH}\right.$ of purine ring). ${ }^{13} \mathrm{C} \mathrm{NMR}(400 \mathrm{MHz}$, DMSO), 74.53 (C-5), 113.26 (C-9), 113.59 (C-8), 122.92 (C-13, C-14), 126.51 (C-12, C-15), 129.39 (C-11), 131.78 (C-10) 157.23 (C-7), 162.66 (C-4, C-6), 168.62 (C-2) $\delta$ ppm. FTIR (KBr) $v_{\max } \mathrm{cm}^{-1}: 1215$ ( $\mathrm{m},-\mathrm{O}-\mathrm{C}$ stretching), 1362 (s, C-NH, aromatic amine), 1632 (s, =C-NH aliphatic amine), 1698 (s, C=O), 2821 (exocyclic CH), 3076 (m, CH-NH stretching), 3342 (b, COH). $\lambda$ max: $291.03 \mathrm{~nm}$; $\left(€: 0.95 \times 10^{5} \mathrm{~L} \mathrm{~mol}^{-1} \mathrm{~cm}^{-1}\right)$; M.W. 305.27, ESIMS: $m / z 306.29(\mathrm{M}+1)$; Anal. Calcd. $\mathrm{C}_{10} \mathrm{H}_{7} \mathrm{~N}_{7} \mathrm{O}_{3} \mathrm{~S}$ 
(\%): C 39.34, H 2.31, N 32.12. Found (\%): C 39.31, H 2.35, N 32.09 .

2.2.7. 5-(((2-Hydroxypyrimidin-4-yl)amino)methylene)pyrimidine-2,4,6-(1H,3H,5H)-trione (BC). Yellow powder, yield $82 \%$; m.p. $>250^{\circ} \mathrm{C} ;{ }^{1} \mathrm{H}$ NMR (400 MHz, DMSO), $4.06 \delta$ ppm $(1 \mathrm{H}, \mathrm{dd}$, exocyclic $\mathrm{NH}$ of purine ring, $j=15.68 \mathrm{~Hz})$, $6.98 \delta \mathrm{ppm}(2 \mathrm{H}, \mathrm{s},-\mathrm{C} \underline{\mathrm{H}}$ of cytosine ring), $8.11 \delta \mathrm{ppm}(1 \mathrm{H}$, $\mathrm{dd}$, exocyclic $\mathrm{CH}$ of pyrimidine ring, $j=23.60 \mathrm{~Hz}), 8.16 \delta$ ppm $(1 \mathrm{H}, \mathrm{dd}, \overline{\mathrm{NH}}$ of cytosine ring), $11.18 \delta \mathrm{ppm}(1 \mathrm{H}, \mathrm{s}, \underline{\mathrm{NH}}$ of pyrimidine ring), $11.26 \delta \mathrm{ppm}(1 \mathrm{H}, \mathrm{s}, \mathrm{N} \underline{\mathrm{H}}$ of pyrimidine ring). ${ }^{13} \mathrm{C}$ NMR (400 MHz, DMSO), 78.53 (C-7), 113.26 (C-9), 113.68 (C-8), 121.29 (C-13), 126.43 (C-12, C-14), 129.58 (C-11, C-15), 133.18 (C-10) 157.53 (C-7), 161.35 (C-4, C-6), $169.34(\mathrm{C}-2) \delta$ ppm. FTIR (KBr) $v_{\max } \mathrm{cm}^{-1}: 1209$ (m, -O-C stretching), 1354 (s, C-NH, aromatic amine), 1632 (s, =C-NH aliphatic amine), 1694 ( $\mathrm{s}, \mathrm{C}=\mathrm{O}$ aromatic and $\alpha, \beta$-unsaturated ketone), 3072 (m, CH-NH stretching). $\lambda \max : 286.34 \mathrm{~nm}$; (€: $1.14 \times 10^{5} \mathrm{~L} \mathrm{~mol}^{-1} \mathrm{~cm}^{-1}$ ); M.W. 249.18, ESIMS: $m / z 251.14$ $(\mathrm{M}+2)$; Anal. Calcd. for $\mathrm{C}_{9} \mathrm{H}_{7} \mathrm{~N}_{5} \mathrm{O}_{4}(\%)$ : C 43.38, $\mathrm{H} 2.83, \mathrm{~N}$ 28.11. Found (\%): C 43.40, H 2.79, N 28.12.

2.2.8. 5-((2-Hydroxypyrimidine-4-ylamino)methylene)-dihydro-2-thioxopyrimidine-4,6(1H,5H)-dione (TBC). Dark orange powder, yield $75 \%$; m.p. $>250{ }^{\circ} \mathrm{C} ;{ }^{1} \mathrm{H}$ NMR $(400 \mathrm{MHz}$, DMSO), $4.09 \delta \mathrm{ppm}(1 \mathrm{H}$, dd, exocyclic $\mathrm{NH}$ of purine ring, $j=13.8 \mathrm{~Hz}), 6.95 \delta \mathrm{ppm}(2 \mathrm{H}, \mathrm{s},-\mathrm{CH}$ of cytosine ring), $8.07 \delta \mathrm{ppm}(1 \mathrm{H}, \mathrm{dd}$, exocyclic $\mathrm{CH}$ of pyrimidine ring, $j=23.66 \mathrm{~Hz}), 8.18 \delta \mathrm{ppm}(1 \mathrm{H}, \mathrm{dd}, \mathrm{N} \underline{\mathrm{H}}$ of cytosine ring), 11.12 $\delta \mathrm{ppm}(1 \mathrm{H}, \mathrm{s}, \mathrm{NH}$ of pyrimidine ring), $11.19 \delta \mathrm{ppm}(1 \mathrm{H}, \mathrm{s}, \mathrm{NH}$ of pyrimidine ring). ${ }^{13} \mathrm{C} \mathrm{NMR}(400 \mathrm{MHz}, \mathrm{DMSO}), 77$ (C-5), 113.45 (C-9), 113.76 (C-8), 121.78 (C-13), 126.34 (C-12, C-14), 129.87 (C-11, C-15), 132.22 (C-10), 158.25 (C-7), 166.15 (C-4, C-6), $169.26(\mathrm{C}-2) \delta$ ppm. FTIR $(\mathrm{KBr}) v_{\max } \mathrm{cm}^{-1}: 1213(\mathrm{~m}$, -O-C stretching), 1626 (s, =C-NH aliphatic amine), 1699 (s, $\mathrm{C}=\mathrm{O}$ ), 2809 (exocyclic $\mathrm{CH}$ ), 3078 (m, CH-NH stretching). $\lambda$ max: $284.46 \mathrm{~nm}$; ESIMS: $m / z 284.45(\mathrm{M}) ;(€: 1.07 \times$ $10^{5} \mathrm{~L} \mathrm{~mol}^{-1} \mathrm{~cm}^{-1}$ ); M.W. 265.25, Anal. Calcd. $\mathrm{C}_{9} \mathrm{H}_{7} \mathrm{~N}_{5} \mathrm{O}_{3} \mathrm{~S}$ (\%): C 40.75, H 2.66, N 26.40. Found (\%): C 40.71, H 2.69, N 26.42 .

2.2.9. 5-((1,6-Dihydro-2-hydroxypyrimidine-4-ylamino)methylene)-1,3-dimethylpyrimidine-2,4,6(1H,3H,5H)-trione $(1,3-B C)$. Yellow powder, yield 65\%; m.p. $>250{ }^{\circ} \mathrm{C} ;{ }^{1} \mathrm{H}$ NMR (400 MHz, DMSO), $2.73\left(6 \mathrm{H}, \mathrm{s}\right.$, two $\mathrm{CH}_{3}$ group of pyrimidine ring), $4.07 \delta \mathrm{ppm}(1 \mathrm{H}$, dd, exocyclic $\overline{\mathrm{NH}}$ of purine ring, $j=14.52 \mathrm{~Hz}), 6.92 \delta \mathrm{ppm}(2 \mathrm{H}, \mathrm{s},-\mathrm{CH}$ of cytosine ring), $8.13 \delta \mathrm{ppm}(1 \mathrm{H}, \mathrm{dd}$, exocyclic $\mathrm{CH}$ of pyrimidine ring, $j=23.62 \mathrm{~Hz}), 8.15 \delta \mathrm{ppm}\left(1 \mathrm{H}, \mathrm{dd}, \mathrm{NH}\right.$ of cytosine ring). ${ }^{13} \mathrm{C}$ NMR (400 MHz, DMSO), $26.6\left(\mathrm{~N}-\mathrm{CH}_{3}\right), 35.9$ (C-11), 79.5 (C-5), 96.5 (C-12), 140.9 (C-8), 146.1 (C-7), 162.4 (C-4, C-6), $169.54(\mathrm{C}-10), 172.6(\mathrm{C}-2) \delta \mathrm{ppm}$. FTIR $(\mathrm{KBr}) v_{\max } \mathrm{cm}^{-1}: 1217$ ( $\mathrm{m},-\mathrm{O}-\mathrm{C}$ stretching), 1634 ( $\mathrm{s},=\mathrm{C}-\mathrm{NH}$ aliphatic amine), 1708 (s, $\mathrm{C}=\mathrm{O}), 2809$ ( $\mathrm{m},-\mathrm{N}-\mathrm{CH}_{3}$ stretching), 2824 (exocyclic $\mathrm{CH}$ ), 3082 (m, CH-NH stretching). $\lambda$ max: $292.44 \mathrm{~nm}$; $(€: 1.05 \times$ $\left.10^{5} \mathrm{~L} \mathrm{~mol}^{-1} \mathrm{~cm}^{-1}\right)$; M.W. 277.24, ESIMS: $m / z 278.22(\mathrm{M}+1)$;
Anal. Calcd. $\mathrm{C}_{11} \mathrm{H}_{13} \mathrm{~N}_{5} \mathrm{O}_{4}$ (\%): C 47.31, H 4.69, N 25.08 . Found (\%): C 47.29, H 4.74, N 25.09.

2.2.10. 5-((1,6-Dihydro-2-hydroxypyrimidin-4-ylamino)methylene)-dihydro-1,3-dimethyl-2-thioxopyrimidine-4,6(1H,5H)dione (1,3-TBC). Orange powder, yield $65 \%$; m.p. $>250^{\circ} \mathrm{C}$; ${ }^{1} \mathrm{H}$ NMR (400 MHz, DMSO), 2.78 (6H, s, two $\mathrm{CH}_{3}$ group of pyrimidine ring), $4.04 \delta \mathrm{ppm}(1 \mathrm{H}$, dd, exocyclic $\underline{\mathrm{NH}}$ of purine ring, $j=16.28 \mathrm{~Hz}), 6.89 \delta \mathrm{ppm}(2 \mathrm{H}, \mathrm{s},-\mathrm{CH}$ of cytosine ring), $8.17 \delta \mathrm{ppm}(1 \mathrm{H}, \mathrm{dd}$, exocyclic $\mathrm{CH}$ of pyrimidine ring, $j=24.62 \mathrm{~Hz}), 8.20 \delta \mathrm{ppm}(1 \mathrm{H}, \mathrm{dd}, \mathrm{NH}$ of cytosine ring $) .{ }^{13} \mathrm{C}$ NMR (400 MHz, DMSO), $34.6\left(\mathrm{~N}-\mathrm{CH}_{3}\right), 39.7$ (C-11), 78.8 (C-5), 97.5 (C-12), 139.6 (C-8), 144.5 (C-7), 161.7 (C-4, C-6), $169.54(\mathrm{C}-8), 173.6(\mathrm{C}-2) \delta \mathrm{ppm}$. FTIR $(\mathrm{KBr}) v_{\max } \mathrm{cm}^{-1}: 1212$ ( $\mathrm{m},-\mathrm{O}-\mathrm{C}$ stretching), 1628 ( $\mathrm{s},=\mathrm{C}-\mathrm{NH}$ aliphatic amine), 1691 (s, $\mathrm{C}=\mathrm{O}$ ), 2797 ( $\mathrm{m},-\mathrm{N}-\mathrm{CH}_{3}$ stretching), 2813 (exocyclic $\mathrm{CH}$ ), 3068 (m, CH-NH stretching). $\lambda$ max: $281.17 \mathrm{~nm}$; (€: $0.95 \times$ $\left.10^{5} \mathrm{~L} \mathrm{~mol}^{-1} \mathrm{~cm}^{-1}\right)$; M.W. 293.30, ESIMS: $m / z 295.27(\mathrm{M}+2)$; Anal. Calcd. $\mathrm{C}_{11} \mathrm{H}_{13} \mathrm{~N}_{5} \mathrm{O}_{3} \mathrm{~S}$ (\%): C 44.74, $\mathrm{H}$ 4.44, $\mathrm{N}$ 23.71, Found (\%): C 44.71, H 4.46, N 23.70.

2.2.11. 5-((Pyridin-2-ylamino)methylene)pyrimidine-2,4,6(1H, $3 \mathrm{H}, 5 \mathrm{H})$-trione (2-BAP). Yellow powder, yield 65\%; m.p. $>250^{\circ} \mathrm{C} ;{ }^{1} \mathrm{H}$ NMR (400 MHz, DMSO), $4.05 \delta \mathrm{ppm}(1 \mathrm{H}, \mathrm{dd}$, exocyclic $\mathrm{N} \underline{\mathrm{H}}$ of pyridine ring, $j=16.38 \mathrm{~Hz}), 6.59-8.09$ $\delta \mathrm{ppm}(4 \mathrm{H}, \mathrm{m},-\mathrm{CH}$ of Pyridine ring), $8.23 \delta \mathrm{ppm}(1 \mathrm{H}$, $\mathrm{dd}$, exocyclic $\mathrm{CH}$ of pyrimidine ring, $j=23.68 \mathrm{~Hz}), 10.89$ $\delta \mathrm{ppm}(1 \mathrm{H}, \mathrm{s}, \overline{\mathrm{N}} \underline{\mathrm{H}}$ of pyrimidine ring), $11.21 \delta \mathrm{ppm}(1 \mathrm{H}$, $\mathrm{s}, \mathrm{NH}$ of pyrimidine ring). ${ }^{13} \mathrm{C} \mathrm{NMR}(400 \mathrm{MHz}, \mathrm{DMSO})$, 80.2 (C-5), 108.5 (C-13), 113.7 (C-11), 138.2 (C-12), 147.8, (C10), 151.3 (C-2), 154.4 (C-7), 164.7 (C-4, C-6) $\delta$ ppm. FTIR $(\mathrm{KBr}) v_{\max } \mathrm{cm}^{-1}: 1218(\mathrm{~m},-\mathrm{O}-\mathrm{C}$ stretching), 1622 (=C$\mathrm{NH}$ aliphatic amine), $1697(\mathrm{C}=\mathrm{O}), 2815$ (exocyclic $\mathrm{CH}$ ), 3078 (CH-NH stretching). $\lambda \max : 288.17 \mathrm{~nm}$; $(€: 0.95 \times$ $\left.10^{5} \mathrm{~L} \mathrm{~mol}^{-1} \mathrm{~cm}^{-1}\right)$; M.W. 232.20, ESIMS: $m / z 232.18$ (M); Anal. Calcd. $\mathrm{C}_{10} \mathrm{H}_{8} \mathrm{~N}_{4} \mathrm{O}_{3}$ (\%): C 51.73, $\mathrm{H} 3.47, \mathrm{~N} 24.13$, Found (\%): C 51.74, H 3.45, N 24.15.

2.2.12. 5-((5-Methylthiazol-2-ylamino)methylene)pyrimidine2,4,6(1H,3H,5H)-trione (BAMT). Orange powder, yield 65\%; m.p. $>250^{\circ} \mathrm{C} ;{ }^{1} \mathrm{H}$ NMR (400 MHz, DMSO), 2.39 (3H, s, $-\mathrm{CH}_{3}$ group of thiazol ring), $4.06 \delta \mathrm{ppm}(1 \mathrm{H}$, dd, exocyclic $\underline{\mathrm{NH}}$ of thiazol ring, $j=15.38 \mathrm{~Hz}), 7.17 \delta \mathrm{ppm}(1 \mathrm{H}, \mathrm{s},-\mathrm{CH}$ of thiazol ring), $8.17 \delta \mathrm{ppm}(1 \mathrm{H}$, dd, exocyclic $\mathrm{CH}$ of pyrimidine ring, $j=24.36 \mathrm{~Hz}), 10.96 \delta \mathrm{ppm}(1 \mathrm{H}, \mathrm{s}, \mathrm{N} \underline{\mathrm{H}}$ of pyrimidine ring), $11.26 \delta \mathrm{ppm}$ (1H, s, $\mathrm{NH}$ of pyrimidine ring). ${ }^{13} \mathrm{C} \mathrm{NMR}$ (400 MHz, DMSO), $17.6\left(-\mathrm{CH}_{3}\right), 78.8$ (C-5), 120.7 (C-11), 134.9 (C-12), 150.4 (C-2), 161.7 (C-9), 165.3 (C-4, C-6), 167.5 (C-7) $\delta$ ppm. FT-IR (KBr) $v_{\max } \mathrm{cm}^{-1}: 1215$ (m, -O-C stretching), 1256 (C-S), 1625 (=C-NH aliphatic amine), 1693 (C=O), 2236 (C-N of thiazol ring), 2794 (- $\mathrm{CH}_{3}$ stretching), 2813 (exocyclic $\mathrm{CH}), 3073$ (CH-NH stretching). $\lambda$ max: $281.17 \mathrm{~nm}$; $(€: 0.95 \times$ $\left.10^{5} \mathrm{~L} \mathrm{~mol}^{-1} \mathrm{~cm}^{-1}\right)$; M.W. 252.25, ESIMS: $m / z 253.05(\mathrm{M}+1)$; Anal. Calcd. $\mathrm{C}_{9} \mathrm{H}_{8} \mathrm{~N}_{4} \mathrm{O}_{3} \mathrm{~S}(\%)$ : C 42.85, H 3.20, N 22.21, Found (\%): C 42.82, H 3.21, N 22.23. 


\subsection{Pharmacology}

2.3.1. Biological Evaluation of the Isolated Compounds. DNA (Calf Thymus type 1), bleomycin sulfate, butylated hydroxyanisole, thiobarbituric acid (TBA), ethylenediaminetetraacetic acid (EDTA), and ascorbic acid were obtained from Sigma. 2,2' -azo-bis-(2-amidinopropane) dihydrochloride and ABTS were purchased from Wako Co., USA.

2.3.2. Antioxidant Screening Assay (ABTS Method). 2,2'azino-bis-3-ethylbenzthiazol-ine-6-sulfonic acid (ABTS) was purchased from Wako Co., USA. L-ascorbic acid was obtained from Sigma and all other chemicals were of the highest quality available. For each of the investigated compounds $(2 \mathrm{~mL})$, ABTS solution $(60 \mu \mathrm{M})$ was added to $3 \mathrm{~mL}$ $\mathrm{MnO}_{2}$ solution $(25 \mathrm{mg} / \mathrm{mL})$, all prepared in $(5 \mathrm{~mL})$ aqueous phosphate buffer solution $(\mathrm{pH} 7,0.1 \mathrm{M})$. The mixture was shaken, centrifuged, and filtered and the absorbance of the resulting green-blue solution (ABTS radical solution) at $734 \mathrm{~nm}$ was adjusted to approx. 0.5 . Then, $50 \mu \mathrm{L}$ of $(2 \mathrm{mM})$ solution of the tested compound in spectroscopic grade $\mathrm{MeOH} /$ phosphate buffer $(1: 1)$ was added. The absorbance was measured and the reduction in color intensity was expressed as inhibition percentage. L-ascorbic acid was used as standard antioxidant (positive control). Blank sample was run without ABTS using $\mathrm{MeOH} /$ phosphate buffer $(1: 1)$ instead of tested compounds. Negative control was run with $\mathrm{ABTS}$ and $\mathrm{MeOH} /$ phosphate buffer (1:1) only. The absorbance $A$ (test ( was measured and the reduction in color intensity was expressed as \% inhibition. The inhibition for each compound was calculated from the following equation:

$$
\% \text { inhibition }=\left[\frac{\{A \text { (control })-A \text { (test })\}}{A(\text { control })}\right] \times 100 \text {. }
$$

Ascorbic acid (vitamin C) was used as standard antioxidant (positive control). Blank sample was run without ABTS using methanol/phosphate buffer $(1: 1)$ instead of sample. Negative control sample was run with methanol/phosphate buffer $(1: 1)$ instead of tested compound $[13,14]$.

2.3.3. Antioxidant Activity Screening Assay 2,2'-Azino-bis3ethylbenzthiazoline-6-sulfonic Acid Method. For each of the investigated compounds, $2 \mathrm{~mL}$ of ABTS solution $(60 \mu \mathrm{M})$ was added to $3 \mathrm{~mL} \mathrm{MnO}_{2}$ solution $(25 \mathrm{mg} / \mathrm{mL}$ ), all prepared in $5 \mathrm{~mL}$ aqueous phosphate buffer solution ( $\mathrm{pH}, 7 ; 0.1 \mathrm{M})$. The mixture was shaken, centrifuged, and filtered, and the absorbance of the resulting green-blue solution (ABTS radical solution) at $\lambda 734 \mathrm{~nm}$ was adjusted to approximately ca. 0.5 . Then, $50 \mu \mathrm{L}$ of $(2 \mathrm{mM})$ solution of the tested compound in spectroscopic grade $\mathrm{MeOH} /$ phosphate buffer (1:1) was added. The absorbance was measured and the reduction in color intensity was expressed as inhibition percentage. L-ascorbic acid was used as standard antioxidant (positive control). Blank sample was run without ABTS and using $\mathrm{MeOH} /$ phosphate buffer $(1: 1)$ instead of tested compounds $[15,16]$.

2.3.4. Bleomycin-Dependent DNA Damage Assay. The reaction mixture contained DNA $(0.5 \mathrm{mg} / \mathrm{mL})$, bleomycin sulfate
(0.05 mg/mL), $\mathrm{MgCl}_{2}(5 \mathrm{mM}), \mathrm{FeCl}_{3}(50 \mathrm{mM})$, and samples to be tested in a conc. of $0.1 \mathrm{mg} / \mathrm{mL}$. L-ascorbic acid was used as positive control. The mixture was incubated at $37^{\circ} \mathrm{C}$ for 1 hour. The reaction was terminated by addition of $0.05 \mathrm{~mL}$ EDTA $(0.1 \mathrm{M})$. The color was developed by adding $0.5 \mathrm{~mL}$ TBA ( $1 \% \mathrm{w} / \mathrm{v})$ and $0.5 \mathrm{~mL} \mathrm{HCl}(25 \% \mathrm{v} / \mathrm{v})$, followed by heating at $80^{\circ} \mathrm{C}$ for 10 minutes. After centrifugation, the extent of DNA damage was measured by increase in absorbance at $532 \mathrm{~nm}[16]$.

2.3.5. Lymphocyte Transformation Assay. The viable lymphocytes were adjusted to a concentration of $2 \times 10^{6}$ cells $/ \mathrm{mL}$ in RPMI-1640 medium supplemented with $600 \mu \mathrm{L}$ penicillin, $0.1 \mathrm{~mL}$ streptomycin, $1 \%$ glutamine, 25\% HEPES (N2 hydroxyethylpiperazine-N-D2-ethanesulfonic acid)-buffer, and $20 \%$ fetal calf serum (FCS). The lymphocytes were plated into 96-well tissue culture plates (or Eppendorf tubes). $100 \mu \mathrm{L}$ of the volatile oil solution in DMF $(100 \mu \mathrm{L} / \mathrm{mL})$ and $20 \mu \mathrm{g}$ of the mitogen (PHA) were added to each well. Cell cultures were incubated at $37^{\circ} \mathrm{C}$ in $5 \% \mathrm{CO}_{2}$ atmosphere for $72 \mathrm{~h}$, during which the mitogen produces its maximal effect on DNA synthesis. After culture, 237 cell films were stained by Giemsa stain and average count of percentage of transformed (proliferated) blasts was determined. Aqueous Echinacea purpurea extract (Immulon) and levamisole (Ketrax) were used as positive control (standard immunostimulant), $100 \mu \mathrm{g} / \mathrm{mL}$ of each drug in DMSO [17].

\section{Result and Discussion}

3.1. Chemistry. The synthetic strategy adopted to obtain the target compounds is depicted in Scheme 1. Various nucleic acid bases such as adenine, guanine, cytosine, and heterocyclic amines were refluxed with formic acid in aqueous medium at $60^{\circ} \mathrm{C}$ to yield formamide derivatives of corresponding DNA bases. Thereafter, in situ barbituric acid and its analogs were refluxed giving the corresponding DNA based barbiturates [18].

The important infrared spectral bands and their tentative assignments for DNA-based barbiturates were recorded as $\mathrm{KBr}$ disks and are presented. The IR spectra of synthesized compounds revealed characteristic bands between $2808 \mathrm{~cm}^{-1}$ and $2826 \mathrm{~cm}^{-1}$ confirming the presence of exocyclic $\underline{\mathrm{CH}}$ groups. IR spectrum of the compounds showed characteristic bands between $1691 \mathrm{~cm}^{-1}$ and $1708 \mathrm{~cm}^{-1}$ confirming the presence of $\mathrm{C}=\mathrm{O}$ groups.

${ }^{1} \mathrm{H}$ NMR spectra revealed signals at $3.50 \delta \mathrm{ppm}$ for DMSO solvent, between 4.2 and $4.09 \delta$ ppm for $\mathrm{NH}$ of the DNA base, between 8.03 and $8.17 \delta$ ppm for exocyclic $\mathrm{CH}$ group, and between 8.09 and $11.27 \delta \mathrm{ppm}$ for $\mathrm{NH}$ of pyrimidine ring. From ${ }^{13} \mathrm{C}$ NMR spectra, exocyclic $\mathrm{CH}$ signal was observed between 144.5 and $158.25 \delta \mathrm{ppm}$. The UV absorption spectra were made using DMSO as a solvent in concentrations $\left(10^{-5} \mathrm{M}\right)$. All synthesized new DNA-based barbiturates derivatives showed the strong absorption bands ( $\lambda$ max) in the range $275-305 \mathrm{~nm}$ owing to the $\pi-\pi^{*}$ and $n-\pi^{*}$ transitions as well as presence of chromophoric exocyclic $\mathrm{CH}$ of pyrimidine ring in their UV spectra [19]. 
TABLE 1: Biological activities of compounds towards lymphocyte transformation, ABTS (free radical scavenging), and bleomycin-dependent DNA damage activities.

\begin{tabular}{|c|c|c|c|}
\hline Compound code & $\begin{array}{c}\text { Lymphocyte } \\
\text { transformation assay } \\
\text { at } 50 \mu \mathrm{m}^{\mathrm{a}, \mathrm{b}, \mathrm{g}}\end{array}$ & $\begin{array}{c}\text { ABTS (percentage } \\
\text { of scavenging inhibition) } \text { c,d,e,f,g }^{\text {A }}\end{array}$ & $\begin{array}{c}\text { Bleomycin-dependent } \\
\text { DNA damage assay }{ }^{\mathrm{g}, \mathrm{h}} \\
\text { absorbance } \\
(\lambda \text { max-532) }\end{array}$ \\
\hline Negative control & - & - & - \\
\hline Butylated hydroxyanisole & - & - & 0.0026 \\
\hline Vitamin C (ascorbic acid) & - & - & $0.0038 \pm 0.01$ \\
\hline BA & 19 & $34.3 \pm 0.10$ & $0.210 \pm 1.12$ \\
\hline TBA & 53 & $60.5 \pm 0.00$ & $0.014 \pm 0.15$ \\
\hline $1,3-B A$ & 35 & $41.7 \pm 1.15$ & $0.015 \pm 0.25$ \\
\hline 1,3-TBA & 35 & $25.8 \pm 1.30$ & $0.014 \pm 1.31$ \\
\hline BG & 37 & $49.5 \pm 0.04$ & $0.017 \pm 0.03$ \\
\hline TBG & 45 & $33.2 \pm 0.70$ & $0.016 \pm 0.27$ \\
\hline $\mathrm{BC}$ & 25 & $31.1 \pm 2.38$ & $0.070 \pm 0.20$ \\
\hline ТВC & 68 & $70.8 \pm 0.10$ & $0.011 \pm 0.04$ \\
\hline $1,3-B C$ & 20 & $35.4 \pm 0.24$ & $0.017 \pm 0.05$ \\
\hline $1,3-\mathrm{TBC}$ & 10 & $13.3 \pm 0.14$ & $0.210 \pm 1.18$ \\
\hline 2-BAP & 35 & $30.7 \pm 1.12$ & $0.011 \pm 0.27$ \\
\hline BAMT & 32 & $40.8 \pm 0.10$ & $0.013 \pm 0.05$ \\
\hline
\end{tabular}

${ }^{a}$ Concentration showing $50 \%$ lymphocyte transformation. ${ }^{\mathrm{b}}$ The positive control was Echinacea purpurea (Ech) extract at concentration 50 giving $74 \%$ lymphocyte transformation. ${ }^{\mathrm{c}} \mathrm{ABTS}+$ scavenging activity $(\%)=[(\mathrm{Ac}-\mathrm{As}) / \mathrm{Ac}] \times 100$, where Ac is the absorbance value of the control and As is the absorbance value of the added samples test solution. ${ }^{\mathrm{d}}$ The concentration of the pure compounds was $2 \mathrm{mM}$. ${ }^{\mathrm{e}}$ The concentration showing $50 \%$ inhibition is expressed in $\mathrm{mM}$. ${ }^{\mathrm{f}}$ The positive control was vitamin $\mathrm{C}$ and showed $80.0 \pm 1.04 \% .{ }^{\mathrm{g}}$ Values are means of 3 replicates $\pm \mathrm{SD}$ and showed significant difference at $P<0.05$ by Student's $t$-test. ${ }^{h}$ The positive control was vitamin C $(0.24 \mathrm{mM})$ and showed absorbance $0.0038 \pm 0.01$ at the same concentration of the tested compounds.

\section{Pharmacology}

A variety of DNA base and heterocyclic primary aminesbased barbiturates were tested for antioxidant activity as reflected in the ability to control of ABTS assays. The prooxidant activities of the aforementioned derivatives were assessed by their effects on bleomycin-induced DNA damage. The DNA base and heterocyclic primary amines-based barbiturates manifested potent antioxidative activity in the ABTS assay.

All compounds have been tested to bleomycin-dependent DNA damage. The results indicated that they may have some protective activity to DNA by certain mechanism. A series of compounds (TBC, TBA, BG, 1, 3-BA, and BAMT) exhibited a high antioxidant activity. On the other hand, all compounds show potent protective effect on the DNA from the induced damage by bleomycin with reference to ascorbic acid and butylated hydroxyanisole (Table 1).

Lymphocyte transformation assay involves study of a specific immune response at $50 \mu \mathrm{M}$. The assay investigates the mitogenic effect of synthesized DNA base and heterocyclic primary amines-based barbiturates on T-lymphocytes. The positive control was Echinacea purpurea (Ech) extract at concentration $50 \mu \mathrm{M}$ giving $74 \%$ of lymphocyte transformation. Data obtained from Table 1 indicated compound TBC shows that $68 \%$ lymphocyte transformed at concentration similar to standard while 35-55\% lymphocyte transformed by synthesized barbiturates TBA, TBG, 1,3-BA, 1,3-TBA, BG, and BPA.

Barbiturate is a versatile moiety that exhibits a wide variety of biological activities. Barbiturate moiety acts as "hydrogen binding domain" and "electron donor system." It also acts as a constrained pharmacophore. Many drugs containing barbituric acid nucleus are available on the market such as allobarbital, alphenal, thiopental, and cyclobarbital.

Thioxodihydropyrimidine can act as the bioisosteric replacement of the pyrimidine moiety. Members of this ring system have found their way into such diverse application as pharmaceuticals, oxidation inhibitors, cyanine dyes, and metal complexing agents. Barbiturate derivatives have antimicrobial, anti-inflammatory, anticancer, anticonvulsant, antidepressant, antioxidant, radio protective, and antileishmanial activities. From the obtained results (Table 1), it can be concluded that pyrimidine moiety is essential for the protection activity against DNA damage induced by bleomyciniron complex, and pyrimidine ring is also required for the activity and an unsubstituted phenyl ring exhibited better activity than those substituted derivatives. This good activity of thioxodihydropyrimidine derivatives shows the hypothesis of a direct link between thiol function and an aromatic ring to be a good one. The thiol catches the radical, and afterwards, the aromatic ring permits the trapping of this radical. Moreover, thiobarbituric acid derivative shows a better activity than barbituric acid. 


\section{Conclusion}

In conclusion, we have designed hybrid molecules on the basis of the biological significance of nucleobase and barbituric acid and evaluated their anticancer activities. All of the new synthesized compounds were tested for their protection activity against DNA damage induced by bleomycin-iron complex. Compounds TBC and 2-BAP showed the highest protection activity against DNA damage. On the other hand, compounds BA and 1,3-TBC showed a very low activity, whereas the rest of the tested compounds exhibited well to moderate activity. It can be concluded that barbituric acid moiety is essential for the protection activity against DNA damage induced by bleomycin-iron complex, and thiobarbituric acid ring is also required for the activity. On the other hand, unsubstituted barbituric acid or thiobarbituric acid exhibited better activity than those substituted derivatives, that is, 1,3-dimethyl substituted derivatives. Out of a set of 12 molecules, four compounds TBC, TBA, BG, and TBG exhibit significant antioxidant, bleomycin-dependent DNA damage, and lymphocyte transformation assay activities and could be used as leads for further investigations.

\section{Conflict of Interests}

The authors declare that there is no conflict of interests regarding the publication of this paper.

\section{Authors' Contribution}

All authors have equally contributed to this work.

\section{Acknowledgments}

The authors are very much thankful to the Department of Applied Chemistry, SVNIT, Surat, for providing laboratory facilities and for the financial support by Gujarat Council on Science and Technology (Project no. Gujcost/MRP/202042/12-13/04). For characterization, they are thankful to Mr. D. M. Thumar from Amoli Organic Pvt Ltd., Baroda, Gujarat. Avtarsingh and Manishkumar from SAIF, Panjab University, Chandigarh, India, are gratefully acknowledged.

\section{References}

[1] M. A. Al-Omar, K. M. Youssef, M. A. El-Sherbeny, S. A. Awadalla, and H. I. El-Subbagh, "Synthesis and in vitro antioxidant activity of some new fused pyridine analogs," Archiv der Pharmazie, vol. 338, no. 4, pp. 175-180, 2005.

[2] V. P. Memon and A. R. Sudheer, "Antioxidant and antiinflammatory properties of curcumin," in The Molecular Targets and Therapeutic Uses of Curcumin in Health and Disease, B. B. Aggarwal, Y. Surh, and S. Shishodia, Eds., pp. 106-107, Springer, New York, NY, USA, 2007.

[3] V. B. Djordjevic, "Free radicals in cell biology," International Review of Cytology, vol. 237, pp. 57-89, 2004.

[4] Z. Nace and K. Danijel, "Preparation and reactivity of 5benzylidenebarbituric and 5-benzylidene-2-thiobarbituric acids," Acta Chimica Slovenica, vol. 58, no. 1, pp. 151-157, 2011.
[5] H. S. Basavaraja, K. V. Jayadevaiah, M. M. Hussain, M. M. J. V. Kumar, and P. Basavaraj, "Synthesis of novel piperazine and morpholine linked substituted pyrimidine derivatives as antimicrobial agents," Journal of Pharmaceutical Sciences and Research, vol. 2, no. 1, pp. 5-12, 2010.

[6] A. Holý, I. Votruba, M. Masojídková et al., "6-[2-(Phosphonomethoxy)alkoxy]pyrimidines with antiviral activity," Journal of Medicinal Chemistry, vol. 45, no. 9, pp. 1918-1929, 2002.

[7] P. Andres and A. Marhold, "A new synthesis of 5-trifluoromethyluracil," Journal of Fluorine Chemistry, vol. 77, no. 1, pp. 93-95, 1996.

[8] J. J. Reddick, S. Saha, J.-M. Lee, J. S. Melnick, J. Perkins, and T. P. Begley, "The mechanism of action of bacimethrin, a naturally occurring thiamin antimetabolite," Bioorganic \& Medicinal Chemistry Letters, vol. 11, no. 17, pp. 2245-2248, 2001.

[9] Y. Kashman, S. Carmely, D. Blasberger, S. Hirsch, and D. Green, "Marine natural products: new results from Red Sea invertebrates," Pure and Applied Chemistry, vol. 61, no. 3, pp. 517-520, 1989.

[10] B. D. Dhorajiya, B. S. Bhakhar, and B. Z. Dholakiya, "Synthesis, characterization, solvatochromic properties, and antimicrobial evaluation of 5-acetyl-2-thioxo-dihydro-pyrimidine-4,6dione-based chalcones," Medicinal Chemistry Research, vol. 22, no. 9, pp. 4075-4086, 2013.

[11] J. R. Patel, B. D. Dhorajiya, F. A. Badria, A. S. Ibrahim, and B. Z. Dholakiya, "In-vitro cytotoxicity, antioxidant, bleomycindependent DNA damage and immunomodulatory evaluation of 1-(4-acetylphenyl)-3-aryloxypyrrolidine-2, 5-dione based derivatives," Medicinal Chemistry Research, 2014.

[12] B. D. Dhorajiya and B. Z. Dholakiya, "Green chemistry multicomponent protocol for formylation and Knoevenagel condensation for synthesis of (Z)-5-arylaminomethylene pyrimidine 2, 4, 6-trione derivatives in water," Green Chemistry Letters and Reviews, vol. 7, no. 1, pp. 1-10, 2014.

[13] B. F. Abdel-Wahab, A.-A. S. El-Ahl, and F. A. Badria, "Synthesis of new 2-naphthyl ethers and their protective activities against DNA damage induced by bleomycin-iron," Chemical and Pharmaceutical Bulletin, vol. 57, no. 12, pp. 1348-1351, 2009.

[14] Y. Morimoto, K. Tanaka, Y. Iwakiri, S. Tokuhiro, S. Fukushima, and Y. Takeuchi, "Protective effects of some neutral amino acids against hypotonic hemolysis," Biological \& Pharmaceutical Bulletin, vol. 18, no. 10, pp. 1417-1422, 1995.

[15] E. A. Lissi, B. Modak, R. Torres, J. Escobar, and A. Urzua, “Total antioxidant potential of resinous exudates from Heliotrapium species, and a comparison of the ABTS and DPPH methods," Free Radical Research, vol. 30, no. 6, pp. 471-477, 1999.

[16] A. B. A. El-Gazzar, M. M. Youssef, A. M. S. Youssef, A. A. AbuHashem, and F. A. Badria, "Design and synthesis of azolopyrimidoquinolines, pyrimidoquinazolines as anti-oxidant, antiinflammatory and analgesic activities," European Journal of Medicinal Chemistry, vol. 44, no. 2, pp. 609-624, 2009.

[17] B. R. Mikhaeil, G. T. Maatooq, F. A. Badria, and M. M. A. Amer, "Chemistry and immunomodulatory activity of frankincense oil," Zeitschrift für Naturforschung C, vol. 58, no. 3-4, pp. 230238, 2003.

[18] B. D. Dhorajiya, A. S. Ibrahim, F. A. Badria, and B. Z. Dholakiya, "Design and synthesis of novel nucleobase-based barbiturate derivatives as potential anticancer agents," Medicinal Chemistry Research, vol. 23, no. 2, pp. 839-847, 2014.

[19] R. M. Silverstein and F. X. Webster, Spectrometric Identification of Organic Compounds, John Wiley \& Sons, New York, NY, USA, 6th edition, 1997. 

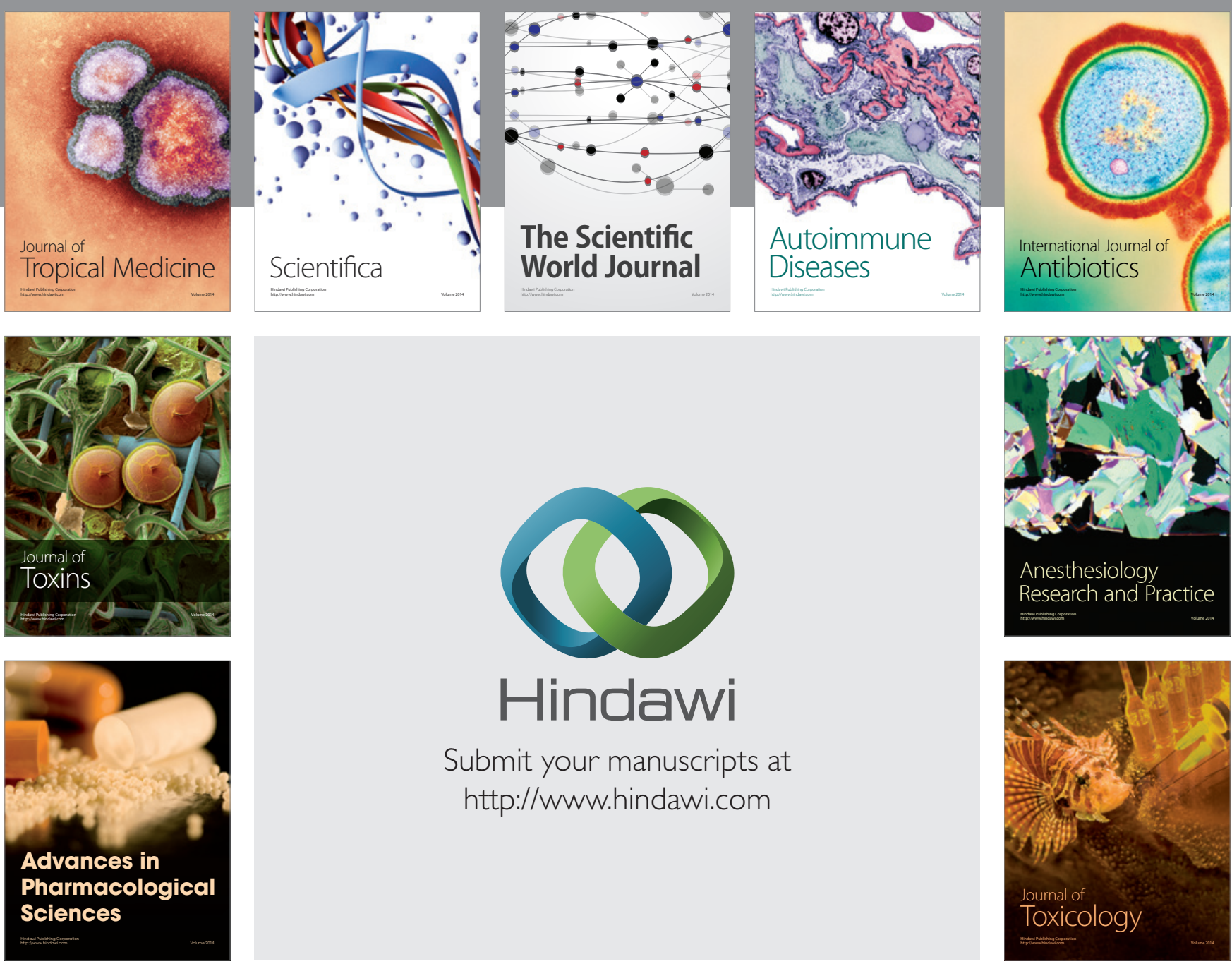

\section{Hindawi}

Submit your manuscripts at

http://www.hindawi.com
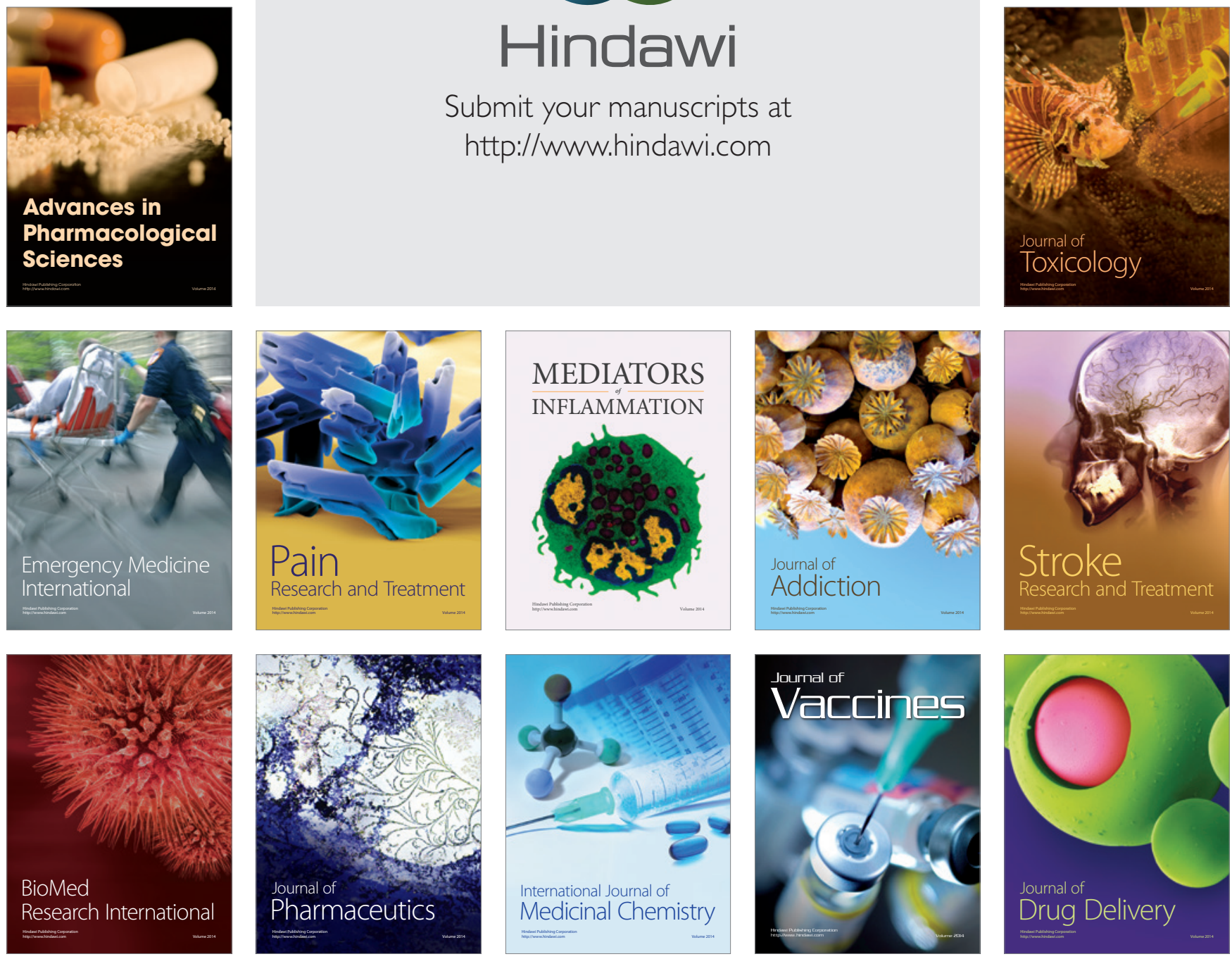